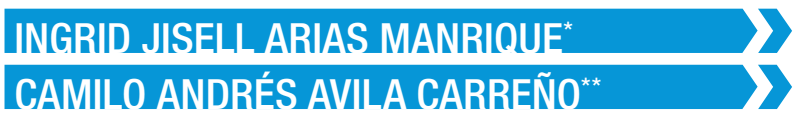

\title{
INFLUENCIA DE LOS PADRES EN EL RENDIMIENTO ACADÉMICO DE LOS HIJOS: UNA APROXIMACIÓN ECONOMETRICA EN EL CONTEXTO DE LA EDUCACIÓN MEDIA COLOMBIANA
}

The influence of parents on their children's academic performance: An econometric approach in the context of Colombian secondary education

A influência dos pais No desempenho acadêmico Dos filhos: Uma abordagem econométrica no contexto do ensino medio colombiano

Este artículo es un derivado del proyecto de investigación titulado: "El Valor Económico de la Educación y Habilidades Cognitivas en los procesos de Desarrollo de Capital Humano", adscrito al grupo de investigación Gestión Organizacional y Desarrollo Humano (GODH) de la Facultad de Ciencias Empresariales de la Universidad de San Buenaventura Bogotá. Investigación dirigía por la Profesora Clara Inés Molina de Barbosa, y bajo la tutoría metodológica de la profesora Jurany Beccie Ramírez.

** Ingrid Jisell Arias Manrique, C.C. 1020794086, Estudiante de IX semestre de Economía de la Universidad de San Buenaventura Bogotá, Colombia. Correo electrónico iarias@academia.usbbog.edu.co. o Jisell_@hotmail.com.

*** Camilo Andrés Avila Carreño, C.C. 10107426969, Estudiante de IX semestre de Economía de la Universidad de San Buenaventura Bogotá, Colombia. Correo electrónico cavila@academia.usbbog.edu.co o Camilo-avila@hotmail.com 


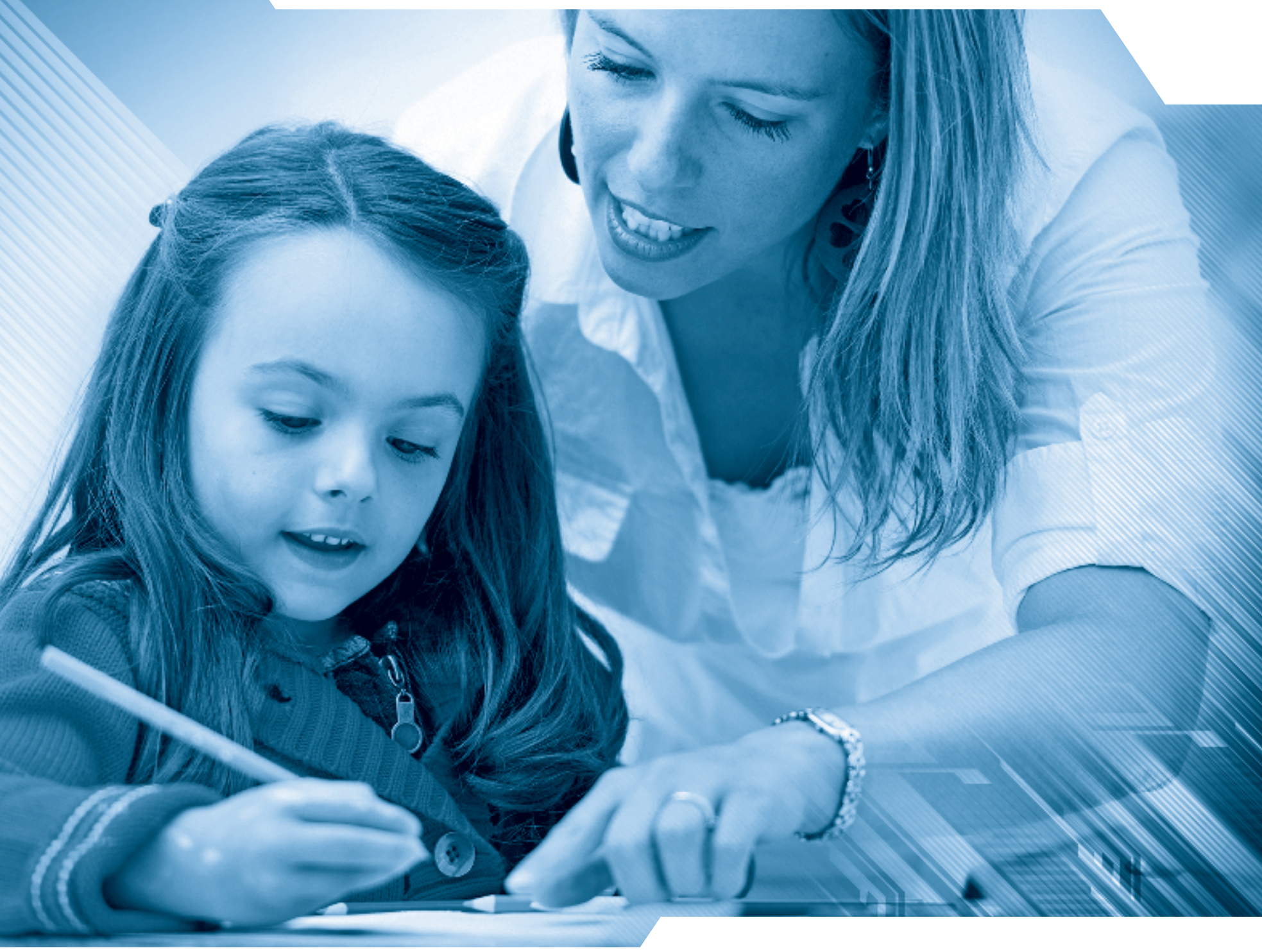

\section{RESUMMEN}

Desde diferentes disciplinas, se ha intentado encontrar respuesta a las problemáticas educativas generadas en escenarios como el de la Educación Media, de manera que dependiendo de estas se han reconocido factores tanto exógenos como endógenos al mismo proceso de enseñanza-aprendizaje. En este sentido, el presente artículo muestra una lectura en perspectiva econométrica, que intenta constituirse en un aporte para proveer argumentos, a propósito de establecer relaciones existentes entre el desempeño de estudiantes de educación media del sistema educativo colombiano, particularmente desde los resultados mostrados en las pruebas de matemáticas y lenguaje de la prueba Saber Pro 2011, con el nivel educativo de los padres de familia de los estudiantes que realizaron dicha evaluación.

Palabras clave: Educación, saber pro, padres 


\section{ABSTRACT}

Different disciplines have looked for the answer to education problems of secondary education and so depending on those, both exogenous and endogenous factors of the learning-teaching process have been acknowledged. This paper shows an econometric reading aimed at providing arguments for establishing relationships between the academic performance of the students of secondary school in the Colombian education system, with basis on the results on the mathematics and language tests of Saber Pro 2011 Test, with the education level of the parents of the students that took that test.

Key Words: Education, Saber Pro, Parents.

\section{RESUMM}

A partir de diferentes disciplinas têm-se tentado encontrar respostas para os problemas educacionais gerados em cenários, como a Educação Mídia, de modo que, dependendo desses fatores foram reconhecidos tanto exógenos e endógenos ao mesmo processo de ensinança -aprendizagem. Neste sentido, este artigo mostra uma leitura em perspectiva econométrica, tentando tornar-se numa contribuição para fornecer argumentos sobre o estabelecimento de relações entre o desempenho dos estudantes do ensino médio do sistema educacional colombiano, particularmente desde os resultados mostrados nas provas de matemática e linguagem da prova Saber Pro 2011, com o nível educativo dos pais dos alunos que realizaram essa avaliação.

Palavras-chave: Educação, Saber Pro, pais.

Dentro de dicho proceso humanizador intervienen varios agentes. Usualmente, se ha reconocido las figuras del estudiante y del profesor. Sin embargo, se ha dejado un poco de lado el papel que pueden jugar otros agentes, como los padres y su círculo social inmediato. Por ello, el presente trabajo toma como punto de partida para el análisis esta última figura (la de los padres), en relación al rendimiento académico de los estudiantes de la educación media colombiana

Ejercicios de este tipo han determinado un sinnúmero de variables en relación con el rendimiento académico de los de los estudiantes. Es así como el rendimiento escolar, se constituye en 
uno de los objetos de estudio fundamentales a la hora de realizar evaluaciones y análisis sobre la práctica y políticas que regulan el proceso de enseñanza-aprendizaje, al integrar un universo de circunstancias y variables, que no necesariamente dependen de los estudiantes y que intentan explicar dicha relación causa-efecto.

No obstante, el hecho de establecer explicaciones alrededor del Rendimiento Académico del estudiante de Educación Media colombiana, dependería a su vez de la concepción que se tenga sobre el concepto mismo de Rendimiento Académico. Por ello, y para los intereses del presente documento, se hace preciso subrayar lo que en adelante se entenderá por dicha categoría, de manera que se pueda entrar en discusión y soportar los resultados a presentar.

En esta dirección, hay que subrayar que el Rendimiento Académico es asumido como la medición cuantitativa de la respuesta del individuo que demuestra lo que ha aprendido durante su proceso de formación académica (Pizzaro \& Clark, 1998).

Esta interpretación en ningún momento busca desestimar otros conceptos existentes, o entrar en discusión con diferentes posturas o perspectivas pedagógicas. Al contrario, pretende demarcar desde el inicio el carácter que toma la lectura que aquí se propone.

Es preciso destacar que otro aspecto fundamental para este ejercicio investigativo, es el rol que cumplen los padres, y más aún, el nivel educativo de éstos, en la explicación del Rendimiento Académico. Así las cosas, se puede afirmar que:

...la educación de los padres es considerada como un factor muy influyente en la determinación del rendimiento académico, así como generalmente a mayor nivel educativo de los padres, mayor es el tiempo dedicado a los hijos y además mayor la calidad de la supervisión al mismo (Martínez et al., s.f., p. 4).

De otra parte, se puede reconocer también que el nivel educativo de la madre, a quien usualmente se la atribuido mayor responsabilidad en la formación de los hijos, puede ligarse con el rendimiento académico del hijo a partir de una relación positiva ${ }^{1}$, lo cual enmarca una realidad social en la cual la mujer dentro del hogar es la encargada del cuidado de sus hijos y de la educación de los mismos (Murnane, Maynard, \& Ohls, 1981).

Como antecedentes investigativos, se reconocen los trabajos de Hakkinen et al, (2003) y Woessmann (2003), citados por Nieto \& Ramos (2011), quienes aseveran que los estudiantes cuyos padres disponen de un nivel de estudio mayor, obtienen un rendimiento académico superior frente a los estudiantes cuyos padres disponen de un menor nivel de estudios. Estas investigaciones han reconocido que una mayor educación de los padres asume un mayor compromiso

1. Una relación lineal (correlación) positiva, es la asociación entre dos variables cuantitativas las cuales se comportan de igual manera o de manera proporcional. 
y generación de hábitos sobre los estudiantes; así mismo, esto ayuda a que el estudiante se centre en sus labores académicas, sin tener que descuidarlas por los percances económicos que puedan surgir. Se afirma además que a un mayor nivel educativo, mayor será la posibilidad de obtener un trabajo estable y seguro que garantice el cumplimiento de los compromisos y, en consecuencia, sea perceptible la importancia de la educación por parte de los padres.

En esta misma dirección, Treimn y Yip en 1989 realizaron un estudio comparativo entre 19 países, con sistemas educativos diversos, tomando en cuenta los años de educación completados de los padres, como las variables dependientes, Comprobaron que en estos países existía una relación positiva entre los años de educación que los padres habían completado y el rendimiento presentado por sus hijos durante su educación. De esta manera, pudieron demostrar que en los países observados, la educación de los padres tiene una influencia positiva sobre la educación del hijo, siendo el resultado extensivo sin importar las diferencias en sus métodos de formación académica (Pérez, Rodríguez \& Fernández, 2009).

De acuerdo a lo anterior, y a los elementos aportados por la perspectiva econométrica, tales como una visión objetiva de los fenómenos, cuantificación de la relación entre las variables y las probabilidades de ocurrencias de los fenómenos, a continuación se realizará una lectura a la relación existente entre el Rendimiento Académico de los estudiantes colombianos de la Educación media, a partir de los resultados arrojados por la Prueba Saber Pro del año 2011, con el nivel educativo de los padres de familia de los estudiantes que realizaron dicha evaluación.

Así las cosas, el presente artículo intenta avanzar más allá del hecho de determinar el tipo de relación entre la educación de los padres con el Rendimiento Académico de sus hijos en el contexto colombiano, sea ésta positiva o negativa, para poder ofrecer grados cuantificables en la relación de las variables que intervienen en el estudio que aquí se propone.

El nivel educativo de la madre, a quien usualmente se la atribuido mayor responsabilidad en la formación de los hijos, puede ligarse con el rendimiento académico del hijo a partir de una relación positiva. 


\section{MÉTODO}

El presente estudio se llevó a cabo bajo un enfoque metodológico cuantitativo. Si bien se reconoce la dificultad de analizar variables que usualmente han sido leídas en perspectiva cualitativa (tales como género, educación o el uso de recursos tecnológicos y virtuales), no se limita en el uso de estos recursos y estrategias metodológicas, todas ellas provenientes del marco de acción provisto por la econometría y la estadística, para establecer una lectura variables influyentes en el Rendimiento Académico de los estudiantes colombianos durante la Prueba Saber Pro 11, particularmente en las áreas de Lenguaje y Matemáticas.

La manera en la cual se encontraron las correlaciones de las variables incluidas en el estudio, las cuales se explican a continuación, fue por medio de un modelo econométrico en función de logística de probabilidad (Logit). Cabe aclarar que la econometría es la aplicación de diferentes métodos matemáticos y estadísticos en unidades de observaciones, que representan variables. Así, Logit es un método empírico para la comprobación de teorías, en el que se modela la interrelación de las variables y el resultado se expresa en la afección promedio de las variables independientes a las dependientes (Baronio \& Vianco, 2010).

Los modelos econométricos buscan ser una representación de la realidad; por lo tanto, el modelo Logit es la mejor manera de representar la función de probabilidad que busca explicar cómo la educación de los padres afecta el rendimiento académico de los estudiantes que presentaron las pruebas saber pro 11 en el año 2011. modelo Logit muestra la relación de las variables en términos de probabilidad de ocurrencia de un evento, en el que 1 es el nivel máximo alcanzado y representa una probabilidad de ocurrencia del 100\%, mientras que 0 referirá a la no ocurrencia del evento esperado. La expresión matemática original de este modelo es:

$$
L_{i}=\left(\frac{\pi_{k}}{1-\pi_{k}}\right)=Z_{i}
$$

Donde:

$L_{i}=$ Es la razón de chances $^{2}$ en el i-enésimo individuo entre las variables dependientes e independientes.

$\pi_{k}=$ Es la probabilidad de la ocurrencia del evento en el i-enésimo y se encuentra entre 0 y 1.

$Z_{i}=$ Es la equivalencia a las variables independientes y sus respectivos betas en el i-enésimo individuo.

Dado que el presente estudio no reflejará sus resultados como una razón de chance, sino como una probabilidad, se realizaron unas transformaciones matemáticas a la fórmula original,

2. "es una razón, que deriva del cálculo de la posibilidad de los casos dividido entre la posibilidad de los controles" (Carvajal, Peña, Muñoz, Comunián, \& Peña, 2006) 
con la idea de buscar únicamente la probabilidad de ocurrencia de los eventos (Gujarati, 2004). Por lo anterior, este es la expresión:

\section{$\pi_{k}=\frac{e^{z_{1}}}{1-e^{z_{1}}}$}

Donde:

$\pi_{k}=$ Es la probabilidad de la ocurrencia del evento en el i-enésimo y se encuentra entre 0 y 1.

$Z_{i}=$ Es la equivalencia a las variables independientes y sus respectivos betas en el i-enésimo individuo.

Los datos que se han utilizado para este estudio fueron tomados de la bases de datos del ICFES, las cuales contienen los resultados del Examen de Estado que se presenta al terminar la educación media. Para la elaboración de este documento se tomaron datos poblacionales ${ }^{3}$ y no muestrales, obteniendo un total de 572.153 observaciones tanto para el primer como el segundo semestre del año 2011.

Los datos tomados, provienen específicamente de los resultados en las pruebas de matemáticas y lenguaje, desarrolladas en el año en mención, siendo estos los únicos resultados a los cuales se tuvo acceso durante la labor de auxiliares de investigación, lo que sirvió también para la elección de estos temas.
En el ICFES, los resultados se encuentran entre 0 y 100, distinguiéndose 3 rangos: bajo, menor o igual a 30; medio, de 31 a 70; y alto mayor o igual a 70 (Grupo de medición y evaluación, 2000). Estos rangos se utilizaron para analizar la probabilidad de tener un rendimiento óptimo dentro de estas pruebas. Dadas las variables explicativas, se tomó el rango mayor o igual a 70 como el hecho deseable o el hecho que se espera.

Para los intereses de este estudio, es decir, para cuantificar la relación entre variables, se ha optado por definir de forma concreta los conceptos involucrados dentro de la investigación:

La educación de los padres: Dividida en la educación tanto primaria como secundaria completa o incompleta; la educación, ya sea técnica, tecnológica, profesional y/o postgrado completa o incompleta de cada uno de los padres o la no realización de estudios académicos. Con esta variable se busca observar la influencia que tiene el nivel educativo de los padres sobre el resultado académico en la prueba, y si alguno de los dos presenta mayor relevancia sobre el rendimiento. Se espera que a mayor educación de los padres, mayor sea la probabilidad de obtener un puntaje alto por parte del estudiante. Cabe resaltar que para el análisis de esta variable se unió la educación primaria y secundaria (Primsecun) y la técnica, tecnológica, pregrados y postgrados (tecnopropos); por último, los que no tienen ningún tipo de educación formal. En el gráfico 1, se describe la distribución del máximo nivel educativo hecho por los padres de familia:

3. Hace referencia al total de estudiantes que presentaron el examen. 


\section{MÁXIMO NIVEL EDUCATIVO ALCANZADO}

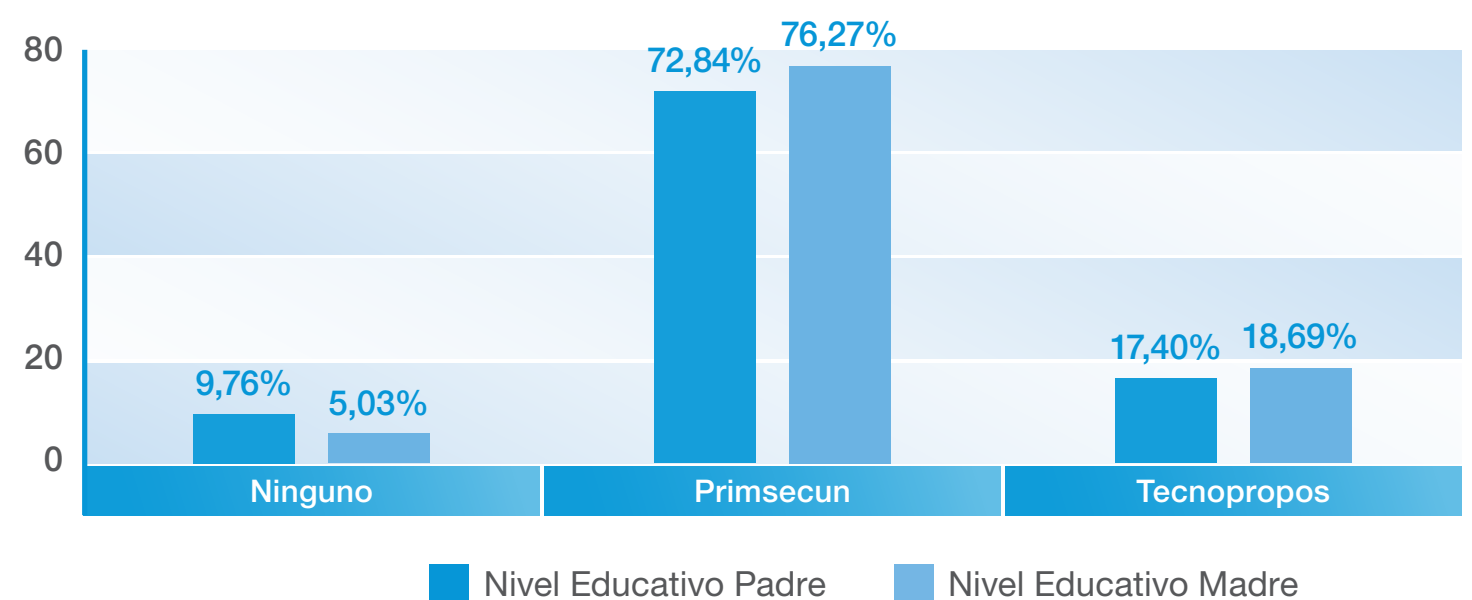

Gráfico 1. Distribución por el máximo nivel de estudios alcanzado de los padres.

Fuente: ICFES. Elaboración propia.

Con referencia a esta variable una descripción estadística de los datos obtenidos muestra que un $72,84 \%$ de los padres han realizado únicamente su educación básica o media, mientras que en este mismo nivel educativo, el 76,27\% madres realizaron estos estudios. En cuanto a los técnicos, tecnólogos, profesional y/o postgrados, las madres son quienes mayor representación tienen (corresponde al 18,69\%), mientras que en los padres el 17,40\% llegó a este nivel educativo. Por último, el 9,76\% de los padres y el 5,03\% de las madres no realizaron ningún estudio.

Internet: Esta variable hace referencia a si en el hogar del estudiante se tiene acceso o no a internet. Se espera que esta variable tenga un efecto positivo en el modelo, dado que es fundamental reconocer que la llegada de la Internet ha cobrado gran importancia en diversas áreas en las que se desempeña el ser humano, y aunque en ocasiones su uso es distorsionado, en el aprendizaje actual es una de las herramientas más utilizadas. Es por ello que el uso en las escuelas esta cada día más presente y va más allá de una herramienta tecnológica: se trata de nuevas metodologías, más dinámicas y atractivas en el proceso de desarrollo del conocimiento.

Es tan significativo el aporte del internet a nuestra vida cotidiana y académica que se ha aseverado que:

Internet se va convirtiendo, inexorablemente, en una especie de tejido nervioso que se va desarrollando rápidamente en nuestras sociedades y se perfila como una herramienta universal para el profesorado de Educación Secundaria en la búsqueda, el intercambio de información, las experiencias formativas y la investigación (López \& Miranda, 2007, p. 52). 


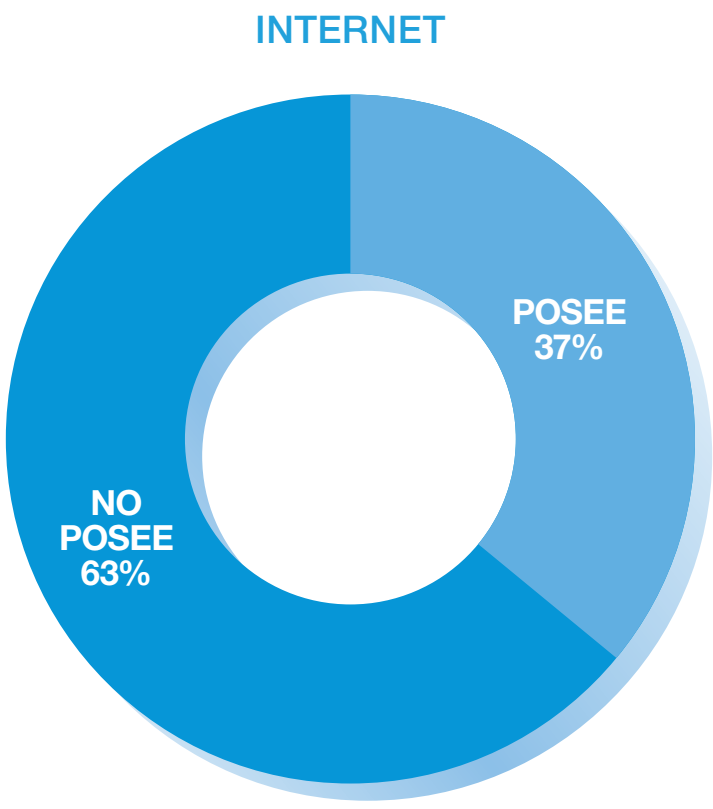

Gráfico 2. Distribución del acceso a internet. Fuente: ICFES. Elaboración propia.

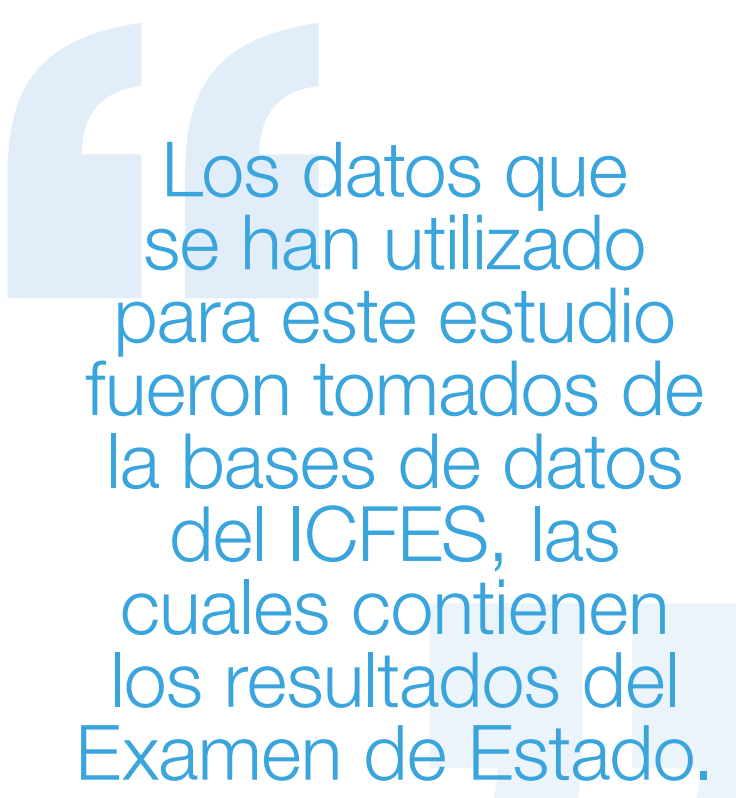

Por lo anterior, se ha tomado en cuenta esta variable dado que aporta de manera significativa al estudio que se ha de realizar, aunque cabe resaltar que esta no será la variable central del estudio.

Como se observa en el gráfico 2, de la población tomada para la elaboración del presente documento se encontró que un 37\% posee internet, al ser una herramienta útil en el proceso de aprendizaje y dadas las facilidades de obtención hoy en día. Aún así hay que tener en cuenta que esto aplica para toda Colombia y que dada la desigualdad que presenta el país no es de extrañar estas cifras presentes.

Género: Se ha tomado esta variable con el fin de observar si existe alguna diferencia entre ser hombre o mujer, en cuanto a los resultados de la prueba. Se espera que esta variable no afecte las probabilidades de obtención de 70 o más en ninguna de las pruebas. Aunque en la educación, la desigualdad de género hoy en día es aun notable, se ha observado que con el pasar de los años las mujeres han obtenido mayor participación y permanencia en las instituciones educativas y que, por el contrario, los hombres tienen una tendencia a disminuir su participación en los últimos años escolares. Pero no basta con su acceso a la educación, se debe presentar también equidad en los resultados y procesos que se llevan a cabo tanto con los hombres como con las mujeres. Como López (2005) afirma: "La idea es que todas las personas, independientemente de su origen social o cultural, deben tener igual acceso al conocimiento. Los sistemas educativos aparecen aquí como igualadores en la formación de los sujetos, como proveedores de los mismos recursos" (López, 2005, p. 71). 


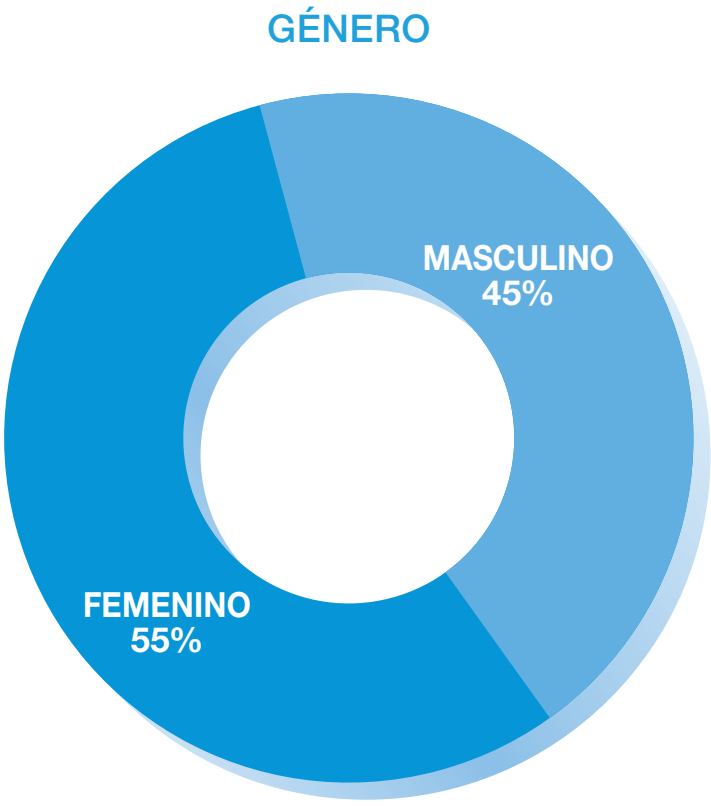

Gráfico 3. Distribución por género.

Fuente: ICFES. Elaboración propia.

En los resultados obtenidos, como se observa en el gráfico 3 , dentro de la población la presencia de mujeres corresponde a un 55,12\% y un $44,88 \%$ son hombres.

De acuerdo a lo anterior, se propone el modelo econométrico que busque explicar la relación entre las variables dependientes e independientes anteriormente descritas, como se expresa a continuación:
Donde:

$\pi_{k}=$ Variable que toma el valor 1 , si el individuo k obtiene un puntaje mayor o igual a 70 en los resultados de las pruebas saber pro 11 en lenguaje matemáticas, siendo estos estudio independiente, y que toma el valor 0 si el individuo k no lo hace.

$\pi_{k}=$ Probabilidad de que el individuo k obtenga un puntaje mayor o igual a 70 en los resultados de las pruebas saber pro 11.

Femeninok2 = Variable que toma el valor 1 , si el individuo $\mathrm{k}$ es mujer y que toma el valor 0 si es hombre.

Primsecun-padre $k 3$ = Variable que toma el valor 1 , si el padre del individuo k cursó primaria y secundaria ya sea completa o incompleta, y que toma el valor 0 en otro caso.

Tecnopropos-padre $k 4$ = Variable que toma el valor 1 , si el padre del individuo k realizó algún técnico, tecnológico, profesional y/o postgrado, ya sea completo o incompleto, y que toma el valor 0 en otro caso.

Primsecun-madre $k 5$ = Variable que toma el valor 1 , si la madre del individuo k cursó primaria y secundaria ya sea completo o incompleto, y que toma el valor 0 en otro caso.

$$
\begin{gathered}
\pi_{k} \sim \operatorname{Bernoulli}\left(\pi_{k}\right) \\
\pi_{k}=\frac{e^{\beta_{1}+\delta_{2} X_{k 2}+\delta_{3} X_{k 3}+\delta_{4} X_{k 4}+\delta_{5} X_{k 5}+\delta_{6} X_{k 6}+\delta_{7} X_{k 7}+u_{k}}}{1-e^{\beta_{1}+\delta_{2} X_{k 2}+\delta_{3} X_{k 3}+\delta_{4} X_{k 4}+\delta_{5} X_{k 5}+\delta_{6} X_{k 6}+\delta_{7} X_{k 7}+u_{k}}} \\
Y_{1}, Y_{2}, \ldots, Y_{n} \text { son independientes }
\end{gathered}
$$


Tecnopropos-madre $k 6=$ Variable que toma el valor 1, si la madre del individuo k realizó algún técnico, tecnológico, profesional y/o postgrado ya sea completo o incompleto, y que toma el valor 0 en otro caso.

Internet $_{k} 7=$ Variable que toma el valor 1 , si el individuo k posee internet, y que toma el valor 0 si no lo posee.

$U_{k}=$ Es el componente aleatorio, que encierra las variables no explicables en el modelo.

Dada la especificación del modelo es de aclarar que el rendimiento de los estudiantes en las pruebas de lenguaje será explicado por las variables de género, educación de los padres e internet, y de manera independiente se analiza la misma relación de estas variables, pero con la prueba de matemática. Es así como se hace necesario la realización de dos regresiones diferentes, una para cada una de las pruebas propuestas y cada uno con sus variables explicativas respectivas.

\section{RESUULTADOS Y ANÁLISIS}

Dado que se opta por observar el Rendimiento Académico a partir de dos pruebas específicas, a saber, lenguaje y matemáticas, este estudio se llevó a partir de dos regresiones. En principio se muestran los resultados de la prueba de lenguaje y posteriormente los de matemáticas. La obtención de los resultados de la regresión econométrica fueron obtenidos gracias al programa estadístico Stata 11.1 SE.

\section{PRUEBALENGUAJE}

La tabla 1 muestra los resultados obtenidos en la regresión con la variable dependiente (prueba de lenguaje), a partir de esto se deduce que en promedio y manteniendo todo lo demás constante, la probabilidad de obtener un puntaje mayor o igual a 70 en la prueba de lenguaje es de un $0,517796 \%$, cuando todas las variables

\section{Tabla 1. Resultado regresión prueba de lenguaje.}

\section{PRUEBA DE LENGUAJE}

\begin{tabular}{|c|c|c|c|}
\hline Significancia global & 0,00 & \\
\hline Variable & Beta & Error Estándar & Significancia \\
\hline Femenino & $-0,000254$ & 0,00016 & 0,109 \\
\hline Primsecun Padre & $-0,000173$ & 0,0004 & 0,667 \\
\hline Tecnopropos Padre & 0,0063042 & 0,0076 & 0,00 \\
\hline Primsecun Madre & 0,0021295 & 0,0053 & 0,00 \\
\hline Tecnopropos Madre & 0,0121588 & 0,00181 & 0,00 \\
\hline Internet & 0,004767 & 0,00024 & 0,00 \\
\hline
\end{tabular}




\section{El acceso a internet también es una variable que afecta positivamente el rendimiento académico, dado que el poseer este servicio aumenta la probabilidad de obtener un puntaje superior a 70 en la prueba de lenguaje.}

independientes son 0, o sea, cuando se es hombre, los padres no han tenido ningún estudio y además no se tiene acceso a internet. Así que entre mil estudiantes que presenten estas características, en promedio, solo 5 estudiantes obtuvieron más de 70 en la prueba de lenguaje.

En cuanto al género, la diferencia entre hombre y mujer no es una variable estadísticamente significativa, con un 95\% de confianza; por lo tanto, inferir acerca de la diferencia en la probabilidad existente entre hombres y mujeres no sería válido. Se puede afirmar que, en este caso, el género no afecta de manera significativa el rendimiento de los estudiantes en el saber pro 11 de 2011.

Cuando el padre tiene algun grado de educación, ya sea primaria o secundaria, la probabilidad disminuye un 0,01726\% lo que lleva a decir que esta variable no es significativa y, en consecuencia, dar cualquier afirmacion sobre esta variable no sería estadisticamente válido. Se puede afirmar que la educación primaria o secundaria en el padre no afectan el rendimiento del estudiante. Sin embargo, cuando la madre tiene ese mismo nivel de educación, la probabilidad del estudiante aumenta en un 0,21295\% y este si es una variable significativa dentro del estudio. Si el padre tiene alguna educación mayor a la secundaria, la probabilidad aumenta en un 0,63042\%, y, para el caso de la madre, la probabilidad aumenta en un 1,21588\% siendo ambas variables significativas. Así, en este caso es evidente que la educación o no de la madre es la que afecta en mayor medida el rendimiento del estudiante, lo que demuestra lo fundamental del vínculo madre e hijo dentro de la formación del estudiante.

El acceso a internet también es una variable que afecta positivamente el rendimiento académico, dado que el poseer este servicio aumenta la probabilidad de obtener un puntaje superior a 70 en la prueba de lenguaje (con un 0,44767\%), siendo ademas una variable significativa dentro del modelo. 


\section{PRUEBA MATEMÁTIIGAS}

A partir de esto se deduce que en promedio y manteniendo todo lo demás constante, la probabilidad de obtener un puntaje mayor $\mathrm{o}$ igual a 70 en la prueba de matemáticas es de un $1,051537 \%$ cuando todas las variables independientes son 0; es decir, cuando se es hombre, los padres no recibieron ninguna educación y además no se tiene acceso a internet. La tabla 2 refleja el resultado de esta progresión:
0,85199\%. Se puede concluir que esta es una diferencia más que considerable si se observa cuánto afectan las demás variables.

Cuando el padre tiene una educación ya sea primaria o secundaria, la probabilidad promedio aumenta un $0,11357 \%$, pero nuevamente esta variable no es significativa dentro del modelo, así que no afecta la probabilidad de obtención del resultado ya mencionado en las prubas Saber Pro 11. Por otro lado, cuando la madre tiene ese

\section{Tabla 2. Resultado regresión prueba de Matemáticas.}

\section{PRUEBA DE MATEMÁTICAS}

\begin{tabular}{|c|c|c|c|}
\hline Significancia global & 0,00 & & \\
\hline Variable & Beta & Error Estandar & 0,00 \\
\hline Femenino & $-0,00852$ & 0,00026 & 0,036 \\
\hline Primsecun Padre & 0,0011357 & 0,0054 & 0,00 \\
\hline Tecnopropos Padre & 0,0134422 & 0,00112 & 0,00 \\
\hline Primsecun Madre & 0,0031832 & 0,00075 & 0,00 \\
\hline Tecnopropos Madre & 0,0188658 & 0,00203 & 0,00 \\
\hline
\end{tabular}

La variable género dentro de esta regresión sí posee un valor significativo (a diferencia de la prueba de lenguaje), por lo cual es válido afirmar que, en promedio y teniendo todas las demás variables constantes, ser mujer disminuye su probabilidad promedio de obtener un resultado mayor a 70 en la prueba de matemáticas un mismo nivel de educación, la probabilidad promedio del estudiante aumenta en un 0,31832\%. Así mismo, si el padre tiene alguna educación mayor a la secundaria, la probabilidad aumenta en un $1,34422 \%$, y si es para el caso de la madre la probabilidad aumenta en un 1,88658\%. Estas últimas tres variables significativas dentro del modelo. 
Por ultimo, el poseer internet dentro del hogar también es una variable significativa y en promedio el acceso a este recurso aumenta las probabilidades en promedio un 0,98964\% de obtener una calificacion mayor a 70 dentro de la prueba de matemáticas.

\section{CONCLUUSIONES}

Lo primero que se puede observar es que estas dos regresiones son significativas globalmente al 95\%, lo que se convierte en un indicio lo suficientemente veraz para afirmar que la especificación del modelo fue realizada de una manera correcta, tanto en la prueba de matemáticas como en la prueba de lenguaje. Además, que sus resultados reflejan de una manera óptima las interrelaciones de las variables sujetas a estudio.

El factor género no es significativo para la prueba de lenguaje, por lo tanto, el ser hombre o mujer no afecta en las probabilidades de sacar más de 70 en esta prueba. Por otro lado, en la prueba de matemáticas sí es significativa la diferencia entre ser hombre y mujer, específicamente se puede afirmar que ser de género femenino reduce la probabilidad de sacar 70 en matemáticas un 0,85199\%.

En cuanto al resultado de primaria y secundaria del padre este no es significativo en ninguna de las dos regresiones. Una de las causas aparentes de este fenómeno, es la baja influencia o la no presencia de los padres dentro de la educación de sus hijos, además del alto nivel en los resultados que se escogió para el estudio. Por lo tanto, es necesario que el nivel educativo del padre sea más alto para influir en el rendimiento educativo de su o sus hijos.
El factor género

no es significativo para la prueba de lenguaje, por lo tanto, el ser hombre o mujer no afecta en las probabilidades de sacar más de 70 en esta prueba. Por otro lado, en la prueba de matemáticas sí es significativa la diferencia entre ser hombre y mujer. 


\section{En cuanto al resultado de primaria}

y secundaria del padre este no es significativo en ninguna de las dos regresiones. Una de las causas aparentes de este fenómeno, es la baja influencia o la no presencia de los padres dentro de la educación de sus hijos.

Como se esperaba, la influencia de la madre es mucho mayor y significativa, dado que es ella quien en muchos de los casos tiene mayor responsabilidad sobre la educación del hijo, aunque también se evidencia que el mayor nivel educativo de la madre afecta en mayor medida y de manera positiva el rendimiento del hijo o hijos en esta prueba. Así, se puede afirmar que en general, a un mayor nivel educativo de los padres mayor será la probabilidad del estudiante de obtener un puntaje alto.

En cuanto a la variable internet, se observó que el tener acceso tuvo una influencia positiva en las dos pruebas, al ser significativas para ambas, si bien difieren en la magnitud de la afección a la variable independiente. Es así como la variable internet aumenta las probabilidades de obtener 70 en la prueba de lenguaje un 0,44767\% en promedio, mientras que la probabilidad en la prueba de matemáticas es en promedio un 0,98964\%. A pesar de demostrar que es un factor importante, también es preocupante el hecho que menos de la mitad de la población aquí estudiada tengan acceso a este recurso.

En conclusión se logra demostrar que el nivel educativo de los padres aumentó las probabilidades de obtener un puntaje mayor o igual a 70 en las pruebas de lenguaje y de matemáticas. A pesar de las bajas probabilidades arrojadas por el estudio, es claro tener en cuenta que el puntaje que se exige es muy superior al promedio que presentó para ese periodo, el cual fue de 52, aproximadamente. De igual manera, las probabilidades de sacar dicho puntaje en la prueba de matemáticas son más altas que las de lenguaje, probablemente por la mayor importancia que se le da a este en los planteles educativos, y bajo el supuesto de que leer implica una detallada comprensión de lectura y otras habilidades que en muchos casos no son adquiridas de buena manera dentro de los planteles de educación básica y media. 


\section{REFERENICIAS}

DBARONIO, A., \& VIANCO, A. (2010). Manual de ECOnometría 1a Parte. Rio Cuarto: Universidad Nacional de Rio Cuarto.

\CARVAJAL, A., PEÑA, G., MUÑOZ, S., COMUNIÁN, G., \& PEÑA, A. (2006). Significado de la razón de posibilidades. Gaceta Médica de Caracas, 114(1),

DDELORS, J. (1996). La Educación encierra un tesoro. Madrid: Santillana S.A.

D) Grupo de medición y evaluación. (2000). Marco de interpretación de resultados. Bogotá: ICFES.

D) GUJARATI, D. (2004). Econometría. Mexico D.F.: McGraw Hill.

》) LÓPEZ, E., \& MIRANDA, M. (2007). Influencia de la tecnología de la información en el rol del profesorado y en los procesos de enseñanza-aprendizaje. RIED, 19(1), 51-60.

\) LÓPEZ, N. (2005). Equidad educativa y desigualdad social: desafios de la educación en el nuevo escenario latinoamericano. Buenos Aires: IIPE-UNESCO.

》) MARTINEZ, C., RÚA, A., REDONDO, R., FABRA, M., NUÑEZ, A., \& MARTÍN, M. (s.f.). Influencia del nivel educativo de los padres en el rendimiento educativo de los estudiantes de ADE. Un enfoque de género. Madrid: Universidad Pontificia Comillas.

DMURNANE, R., MAYNARD, R., \& OHLS, J. (Agosto de 1981). Home Resourses and Children's Archievement: Politicalscience. Obtenido de Political science: http:// www.politicalscience.uncc.edu/godwink/PPOL8687/ WK5\%20Feb\%208\%20Test\%20score\%20gap\%20I/ Murnane\%20Home\%2OInputs\%20and\%20Outcomes\%20among\%20low\%20income\%20families.pdf+

》NIETO, S., \& RAMOS, R. (2011). ¿La sobreeducación de los padres afecta el rendimiento académico de sus hijos? Barcelona: UB IREA.

D) PÉREZ, V., RODRíGUEZ, J. C., \& FERNÁNDEZ, J. J. (2009). Educación y familia. Los padres ante la educación general de sus hijos en España. Madrid: Fundación de las cajas de ahorros.

》PIZZARO, R., \& CLARK, S. (1998). Currículo del Hogar y aprendisajes educativos: Interacción versus Status. Revista de Psicología de la Universidad de Chile, 7, 25-34.

DSAVATER, F. (1997). El valor de educar. Barcelona: Ariel. 